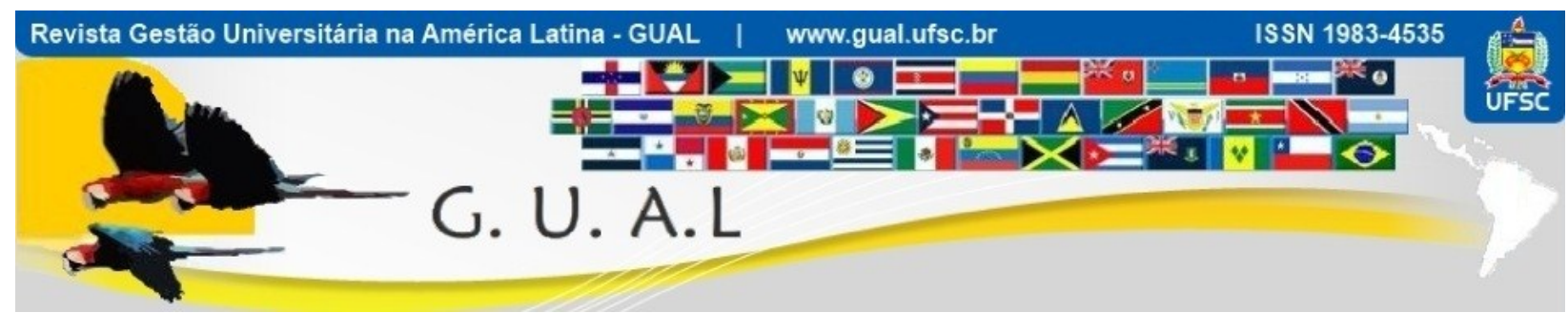

DOI: http://dx.doi.org/10.5007/1983-4535.2012v5n4p68

\title{
EL PROCESO DE ACREDITACIÓN INSTITUCIONAL DEL PE DE PEDAGOGÍA POR EL CEPPE
}

\section{THE PROCESS OF INSTITUTIONAL ACCREDITATION OF TEACHING BY PEI CEPPE}

Areli Pérez Blanco, Mestre Universidad Veracruzana - UV arenita10081@hotmail.com

Brenda González Barraza, Doutor Universidad Veracruzana - UV american_girl22@hotmail.com

Recebido em 16/novembro/2012

Aprovado em 07/dezembro/2012

Sistema de Avaliação: Double Blind Review

Esta obra está sob uma Licença Creative Commons Atribuição-Uso. 


\title{
RESUMEN
}

El presente trabajo trata de la experiencia que tuvieron tanto alumnos como personal docente de la acreditación institucional CEPPE en la Facultad de Pedagogía Región Poza Rica Tuxpan, partiremos desde la fundación de la Facultad de Pedagogía, enfatizando y describiendo todo el proceso de acreditación CEPPE.La visión que se tuvo acerca de la Evaluación que se llevó dentro de la Facultad de Pedagogía particularmente con el Proceso de Acreditación, es que la evaluación es la mejor herramienta para conocer la realidad de una Institución, de un Programa Académico. Esta investigación estuvo sustentada por el Paradigma Cualitativo. Nuestra metodología se inclina a la recolección de datos de manera verbal o escrita y comprender las conductas de los actores de estudio tal y como se muestra en la realidad. En esta investigación Cualitativa, pretendemos describir el Proceso de Acreditación en la Facultad de Pedagogía, es decir, saber cómo piensan los sujetos implicados en esta investigación.

Palabras-clave: CEPPE. Acreditación. Certificación. Evaluación. Institucional.

\begin{abstract}
The present work is the experience that had both students as teachers in the Faculty of pedagogy Region Poza Rica Tuxpan CEPPE institutional accreditation, will depart from the foundation of the Faculty of education, emphasizing and describing the entire process of accreditation CEPPE. The vision that had about assessment which led within the Faculty of education, particularly with the accreditation process, is that the evaluation is the best tool to learn about the reality of an institution of an academic program. This research was supported by the qualitative paradigm. Our methodology is inclined to the data collection of verbal or written and understand the behaviours of the actors Studio as shown in the reality. In this qualitative research, we intend to describe the accreditation process in the Faculty of education, i.e. to know how they think the subjects involved in this research.
\end{abstract}

Keywords: CEPPE. Accreditation. Certification. Evaluation. Institutional. 


\section{INTRODUCCIÒN}

La educación superior tiene como finalidad formar profesionales humanistas e intelectuales capaces de responder a las necesidades de la sociedad; ya que como sabemos la sociedad está en constante cambio y es fundamental la actualización de contenidos curriculares, esto se lograra con ayuda de las herramientas de los conocimientos actuales, por tal modo es de gran importancia proponer alternativas de desarrollo acompañadas de ideales establecidos a lo largo de la historia y expresados en las normas legales. La educación superior es responsable de la formación de profesionales capaces de crear conocimiento y de proponer nuevas formas de análisis y nuevas herramientas y relaciones de trabajo en todas las áreas.

Cabe resaltar que no basta con que las Instituciones de Educación Superior formen profesionales, si no también esta formación consiste en crear mayores compromisos, tanto de los estudiantes como de las mismas instituciones, a causa de esto la educación en nuestro País está cada vez más comprometida en establecer y proporcionar una formación educativa de calidad, participando en procesos de evaluación que avalen la calidad de sus servicios, proceso que acontinuación describiremos brevemente.

A lo largo de la década de los 90's surge un proceso llamado Acreditación, el cual es un reconocimiento público que otorga un organismo acreditador a una institución o programa de estudio, mediante evaluaciones tanto internas como externas por los organismos de evaluación correspondientes, basándose en parámetros de calidad internacionales.

\subsection{JUSTIFICACION:}

Nuestro interés por llevar a cabo esta investigación se dio por que como futuros docentes es fundamental conocer este proceso, y el rol que ocupamos en el para participar activamente y todos trabajemos equilibradamente con las personas que forman parte de la institución y así lograr alcanzar los objetivos de manera activa.

Algunos Organismos encargados de la Acreditación de Instituciones de Educación Superior (IES), son: Consejo para la Acreditación de la Educación Superior (COPAES), Asociación Nacional de Universidades e Instituciones de Educación Superior (ANUIES), y Comité para la Evaluación de Programas de Pedagogía y Educación (CEPPE).

Es esencial que todos los programas educativos de la Universidad Veracruzana sean evaluados, y así lograr el objetivo fundamental que es una Acreditación que tiene como 
propósito una mejora continua de su educación y servicios, esta se lleva acabo por organismos externos para lograr una institución educativa de calidad e inteligente donde los recursos personales que forman parte de esta colaboren en su transformación innovando, actualizándose, siendo creativos y estando capacitados para desempeñar diversas funciones, y así lograr una reforma académica de base y dando resultados de los numerosos actos y procesos que intervienen en la evaluación de una institución. Por tal motivo la Facultad de Pedagogía se vio en la necesidad de entrar en los procesos de Evaluación.

Esta investigación estuvo sustentada por el Paradigma Cualitativo. Nuestra metodología se inclina a la recolección de datos de manera verbal o escrita y comprender las conductas de los actores de estudio tal y como se muestra en la realidad. En esta investigación Cualitativa, pretendemos describir el Proceso de Acreditación en la Facultad de Pedagogía, es decir, saber cómo piensan los sujetos implicados en esta investigación.

\subsection{OBJETIVOS}

Analizar las expectativas que tienen docentes y alumnos después de ser Acreditada la Facultad de Pedagogía.

Describir los lineamientos que siguió la Facultad de Pedagogía de acuerdo al organismo encargado de la acreditación CEPPE (Comité para la Evaluación de Programas de Pedagogía y Educación.

Identificar algunas sugerencias de docentes y alumnos hacia la Facultad de Pedagogía después de ser acreditada.

\section{DESARROLLO}

Concepto de evaluación para (Maccario: 1963) se trata de un acto donde debe emitirse un juicio en torno a un conjunto de información e identificar, obtener y proporcionar información útil y descriptiva acerca del valor y el merito de las metas, la planificación, la realización, y el impacto de un objeto determinado, con el fin de servir de guía para la toma de decisiones, solucionar los problemas de responsabilidad y promover la comprensión de los fenómenos implicados y debe tomarse una decisión de acuerdo a los resultados que presente una institución.

\subsection{CONCEPTO DE ACREDITACIÓN}

Para (Van Vugh: 1993) la acreditación es un proceso en el cual un grupo externo juzga el nivel de calidad de uno o más programas específicos de una institución de educación superior, mediante el uso de estándares preestablecidos. 


\subsection{CONCEPTO DE CERTIFICACIÓN}

(ONNCCE 2009) Es un procedimiento por el cual se asegura que un producto, proceso, sistema o servicio se ajusta a las normas, lineamientos o recomendaciones de organismos dedicados a la Normalización Nacional o Internacional.

El Comité para la Evaluación de Programas de Pedagogía y Educación, (CEPPE 2006) como su nombre lo dice es una asociación civil integrada por organismos representativos del área y por expertos en el área de la evaluación institucional.

Sus principales funciones son: aprobar el presupuesto asociado a las actividades, revisar y en su caso, aprobar los estados financieros auditados de la asociación y nombrar a los integrantes del consejo directivo y consejo técnico.

La misión del CEPPE certificar y promover la calidad y la mejora continua de los programas educativos en el área de pedagogía, educación y otras afines a través de ejercicios de evaluación periódica en las instituciones de educación superior públicas y privadas.

Como visión el CEPPE se consolidará como un organismo nacional con liderazgo en procesos de evaluación y acreditación, particularmente para los programas educativos en pedagogía, educación y áreas afines, que opere en el marco de una cultura de calidad, ética y servicio, para transitar hacia la excelencia en la formación de profesionales en el área educativa.

La CEPPE tiene diferentes objetivos que son los siguientes:

- Establecer un proceso permanente de actualización y mejora del proceso de evaluación a través de la revisión periódica de sus métodos e instrumentos de acreditación.

- Establecer relaciones de cooperación con organismos nacionales e internacionales de evaluación, auto-estudio y acreditación que permitan integrar un sistema de homologación y reconocimiento en estas actividades.

- Implementar los procesos de acreditación a través de un padrón nacional de evaluadores, de amplio reconocimiento profesional y de absoluta solvencia moral.

\subsection{MARCO DE REFERENCIA PARA LA ACREDITACIÓN DE PROGRAMAS DE ACUERDO A CEPPE}

El marco de referencia para la acreditación es un documento que establece los lineamientos técnico metodológico para que pueda desarrollarse el proceso de Acreditación de programas educativos. Este marco de referencia alude a conceptos que son esenciales en todo el proceso de Acreditación, los cuales son los siguientes: 
a)Actores del proceso de Acreditación: éstos son los integrantes del CEPPE que participan de manera directa e indirecta en el proceso de evaluación externa de los programas académicos.

b) Objeto de estudio:son cada uno de los programas académicos de licenciatura y posgrado (especialidad, maestría y doctorado), en sus modalidades escolarizado, abierto y a distancia que deciden someterse a procesos de evaluación externa.

c) Categorías de análisis: son rubros a valorar por el CEPPE, los cuales integran un conjunto de indicadores o dimensiones sujetas a análisis.

d) Dimensiones: describen los distintos elementos que conforman a una categoría de análisis.

e) Indicadores: son enunciados que describen los elementos que se analizan de una dimensión.

f) Parámetros de calidad: son los valores ideales o deseables de un indicador, que están establecidos por el CEPPE, en los cuales se evidencian aspectos cuantitativos y/o cualitativos que permiten llevar a un mayor grado de precisión los criterios de calidad.

\subsection{ETAPAS DEL PROCESO DE ACREDITACIÓN SEGÚN CEPPE}

Este proceso de Acreditación está conformado por 4 etapas que deben ser asumidas por el Director del Programa Educativo o Coordinadores del mismo, sus profesores, administrativos, alumnos y por miembros del CEPPE. Las etapas son las siguientes:

ETAPA 1. Previos al trabajo de auto evaluación

ETAPA 2. Autoevaluación

ETAPA 3. Visita del Comité evaluador

ETAPA 4. Resultado de la Acreditación

\subsection{SEGUIMIENTO Y PLAN DE MEJORA}

La Facultad o Escuela evaluada diseña un plan de mejora continua con base en las observaciones y recomendaciones y lo deberá enviar al CEPPE. Posterior a lo anterior, el CEPPE programará visitas de seguimiento. El CEPPE podrá retirar la acreditación a un programa cuando se demuestre que éste ha dejado de cumplir alguno de los criterios de calidad, cuando se haya realizado alguna modificación importante sin notificación alguna a dicho Comité, o bien si no son atendidas las recomendaciones realizadas. De igual forma, la investigación que nosotras realizamos, tiene como uno de los objetivos principales, proponer la elaboración de un Plan de mejorarealizado por la misma Facultad de Pedagogía, tomando en cuenta las sugerencias que se elaborarán a partir de las opiniones de los sujetos 


\section{EL PROCESO DE ACREDITACIÓN INSTITUCIONAL DEL PE DE PEDAGOGÍA POR EL CEPPE DOI: http://dx.doi.org/10.5007/1983-4535.2012v5n4p68}

entrevistados y se que complemente con las observaciones emitidas por el CEPPE, ahora tomando en cuenta la viva voz, es decir las opiniones y expectativas de los sujetos que interactuamos dentro de la misma institución.

\subsection{ANTECEDENTES DE LA FACULTAD DE PEDAGOGÍA POZA RICA}

Desde el inicio de su gestión administrativa y académica la Facultad de Pedagogía ha estado ubicada geográficamente en la misma dirección, situada en la calle 12 \# 215 de la Colonia Cazones, en Poza Rica, Ver., sin embargo, comparten instalaciones con la Facultad de Trabajo Social teniendo un edificio propio en cuanto a las aulas y el área administrativa.

El plan de estudios con que inició funciones la Facultad de Pedagogía fue el que en esos momentos se encontraba vigente en pedagogía Xalapa cuya fecha de diseño y aprobación fue 1977, el cual tenía un enfoque mono teórico fundamentado en las tendencias pedagógicas vigentes, esto es, la tecnología educativa, de la cual se destaca sus bases conductistas, la programación por objetivos, y la evaluación por pruebas objetivas, asimismo predominaba la investigación de corte cuantitativo.

\section{CONCLUSIONES}

La Universidad Veracruzana Facultad de Pedagogía al haber obtenido a través de la acreditación por parte del Comité para la Evaluación de Programas de Pedagogía y Educación, A. C. (CEPPE) comprueba el gran compromiso que se tiene con y para la sociedad cada vez más compleja.

Esto se llevó a cabo cumpliendo con los requisitos establecidos y con las normas de calidad, pero más allá del reconocimiento, demostró en gran medida, la calidad con la cual se elaboran sus programas educativos y el gran entusiasmo que se tiene por ser mejores y mas competentes, buscando siempre la actualización de su plantilla escolar así como todos aquellos elementos que contribuyan con esta noble labor.

\section{RESULTADOS}

En cuanto a los objetivos planteados en nuestra investigación, fueron alcanzados en su totalidad, ya que para iniciar con este trabajo optamos por indagar en el marco de Referencia del CEPPE, para identificar los lineamientos que siguió la Facultad de Pedagogía en el 
proceso de Acreditación, para que a partir de ahí pudiéramos suponer algunas preguntas en las cuáles giraría nuestra investigación.

Una vez teniendo en claro hacía a donde íbamos con esta investigación, decidimos focalizar nuestro tema en las expectativas de los Docentes y Alumnos después de la Acreditación de la Facultad de Pedagogía, tomando como referencia para nuestro instrumento de investigación el Oficio de Recomendaciones emitido por el mismo organismo acreditador.

Después de entrevistar a los sujetos investigados, proseguimos a categorizar e interpretar las respuestas de los sujetos entrevistados, por lo que notamos que algunos de ellos coincidían en las respuestas, pero también hubo casos en los cuales no siempre se encontraban de acuerdo, lo cual resultó interesante porque de esta manera la información se fue enriqueciendo de tal forma que pudimos realizar un listado de sugerencias a la Facultad de Pedagogía para que de esta forma se pueda elaborar un Plan de Mejora que ayude a la institución a solventar las recomendaciones emitidas por el organismo acreditador CEPPE y así lograr la re acreditación.

Dentro de las fortalezas de la Facultad que descubrimos con nuestra investigación, en cuanto a los docentes, es que la mayoría de estos están en constante actualización en cuanto a sus conocimientos, tienen disposición para colaborar con el progreso de nuestra Facultad así como apoyar el desarrollo académico e intelectual de sus alumnos. En cuanto a los estudiantes, podemos rescatar el gran interés y actitud de superación hacia ellos mismos y hacia su institución, están conscientes de que el resultado de la acreditación debió ser de manera conjunta, y como tal todos son responsables del constante progreso que esta pueda y deba tener.

Las principales expectativas que identificamos con nuestra investigación fueron:

Que los beneficios que se obtiene con la acreditación según lo sujetos son Renombre, obtención de un programa educativo de Calidad y validez en el título de Licenciatura, lo que denota que la formación dentro de la Facultad se espera sea cada vez de mejor calidad.

Otra expectativa fue que consideran los entrevistados que existe una buena preparación académica por parte de los docentes de la Facultad de Pedagogía y ahora con la acreditación puede ser aun mejor, y sugieren que también los estudiantes egresados de Pedagogía continúen con su preparación después de concluir su licenciatura proponiendo que la Facultad brinde cursos de formación continua.

Una expectativa de los sujetos que entrevistamos también fue que los estudiantes están interesados en involucrarse en trabajos de investigación con los docentes, así también los 
docentes están interesados en apoyar y pedir la colaboración a los alumnos para continuar o comenzar algún proyecto de investigación para que de esta manera sus educandos también obtengan conocimientos metodológicos, teóricos y prácticos.

$\mathrm{Y}$ por último se espera que se cumplan y atiendan todas las recomendaciones que se emitieron después de que la Facultad de Pedagogía se acreditó para así poder aspirar a la posible Reacreditación o a una acreditación internacional.

Consideramos que nuestro trabajo de investigación es muy importante porque en primera fue una gran satisfacción para nosotras el realizarlo, en segunda lo consideramos un tema de interés que pocos se adentran a investigar y en tercera deseábamos cooperar con la Facultad para que dentro de cinco años logre una re acreditación, si toman en cuenta las sugerencias elaboradas que surgieron de las opiniones de los sujetos entrevistados, los cuales se expresaron sobre lo que piensan acerca de la acreditación de la Facultad así como de lo que esperan de la misma, además se considera que con la ayuda de toda la comunidad universitaria que labora en ella se logre una mejora en la Institución, se reconozca aun más el nivel educativo que tiene y que los procesos educativos sean aún mejores.

\section{REFERENCIAS BIBLIOGRAFÌA}

Mendoza Rojas, Javier. La evaluación y acreditación de la educación superior mexicana: las experiencias de una década. Centro de Estudios sobre la Universidad Nacional Autónoma de México.

Pallán, Figueroa Carlos. (1994) Los procesos de Evaluación y Acreditación de las Instituciones de Educación Superior en México en los últimos años, en Revista de la Educación Superior, Num. 91, ENERO - MARZO.

Pérez, Rocha Manuel. (1996) Evaluación, acreditación y calidad de la Educación Superior, en Revista de la Educación Superior, Num. 98, ABRIL- JUNIO.

Rodríguez Gómez, Gregorio y Col (1.999) Metodología de la Investigación Cualitativa. Editorial Aljibe. Málaga-España.

Rosado, m. (2003). Metodología de investigación y evaluación. México: Trillas

Rosario, Muñoz Víctor Manuel UV. (2006). Acreditación y certificación de la Educación Superior: experiencias, realidades y retos para las IES.

Stake, R. (1998). Investigación con estudio de casos. Madrid: Morata. 


\section{FUENTES ELECTRONICAS:}

Comité Para la Evaluación de Programas de Pedagogía y Educación, consultado en http://www.ceppe.org.mx/, el 1 de Abril del 2011.

Comités interinstitucionales de Evaluación para la Educación Superior, consultado en http://www.ciees.edu.mx/ciees/quesonciees.php, el 4 Abril del 2011.

Consejo para la Acreditación de la Educación Superior, consultado en http://www.copaes.org.mx/, el 30 Abril del 2011.

Antecedentes de la Facultad de Pedagogía Poza Rica, consultado en http://www.uv.mx/pozarica/pedagogía, el 3 de Marzo del 2011.

Definición de evaluación en (maccario) en http://definicion.de/evaluacion/ dice ( maccario).

Dice Van Vugh en definición de acreditación http://www.uned.ac.cr/paa/pdf/Materialesautoev/09.pdf

Definición de certificación en http://www.onncce.org.mx/images/pdf/laboratoriosDic09.pdf (ONNCCE) 